\title{
Purity determining of cultured OECs from olfactory mucosa of rats' pups
}

Monireh Azizi ${ }^{1}$, Mehrdad Bakhtiari ${ }^{2}$, Farhid Farahmand Ghavi ${ }^{3}$, Mojgan Zandi ${ }^{4}$, Mohammad Imani $^{3}$, Mohammad Taghi Joghataei ${ }^{2 *}$

1. Department of Anatomy, Ilam University of Medical Sciences, Ilam , Iran

2. Molecular and Cellular Research Center, Iran University of Medical Sciences, Tehran, Iran

3. Novel Drug Delivery Systems Department, Iran Polymer and Petrochemical Institute, Tehran, Iran

4. Biomaterials Department, Iran Polymer and Petrochemical Institute, Tehran, Iran

*Corresponding author: Tel: +98 2186704569 Fax: +98 2188622689

Address: Molecular and Cellular Research Center, Iran University of Medical Sciences, Tehran, Iran

E-mail: mt.joghataei @yahoo.com

Received; 2015/08/23 revised; 2015/09/7 accepted; 2015/09/12

\section{Abstract}

Introduction: Cell transplantation is one of the main strategies for spinal cord injury repair. As OECs of the olfactory mucosa can be obtained by simple biopsy in all individuals without affecting their smell sensation, OECs considered as a promising candidate for autologous transplantation in the nervous system injury, especially for spinal cord repair. Thereby in the current study OECs were cultured from olfactory mucosa of 7 days old rats' pups and their purity was examined by flow-cytometry after simultaneous double staining for p75 and GFAP markers.

Materials and methods: 7 days old Wistar rats' pups were deeply anesthetized by ketamine / xylazine $(60 / 6 \mathrm{mg} / \mathrm{Kg})$. Then the nasal cavity was opened sagittally and the olfactory mucosa was separated from posterior part of nasal septum and at last OECs were obtained from lamina properia of olfactory mucosa and were cultured. The cultured cells were simultaneously immunolabeled for p75 and GFAP markers and finally purity of cultured cells assessed by flow-cytometry.

Results: cultured OECs demonstrated two different morphologies, a spindle shape Schwannlike and an astrocyte-like OECs with flat sheet-like morphology. In addition, simultaneous immunolabeling for p75 and GFAP markers of OECs exhibited OECs were positive for both markers at the same time. The flow-cytometry results displayed that $87.9 \pm 2.4 \%$ of cells were p75/ GFAP double positive cells, $1.05 \pm 0.4$ only p75 positive and $5.8 \pm 1.5 \%$ were single positive for GFAP.

Conclusion: Purity of cultured OECs in our study is probably more than $87.9 \%$ by flowowing to $\mathrm{p} 75+/ \mathrm{S} 100+$ and GFAP+/S100+ olfactory ensheathing cells were not counted. Thus the culture procedure of this study seems to be a good protocol for OECs purifying and cell therapy in CNS damages.

Keywords: OECs, Cell therapy, Spinal cord injury, Flowcytometry

\section{Introduction}

Spinal cord injury (SCI) is a major cause of disability but there is no universally accepted treatment yet. Recently, cell transplantation, has become one of the most promising therapeutic approaches for SCI, and was strongly examined by numerous investigators. A number of different types of stem cells, neural progenitors, and glial cells have been examined via experimental researches, but several reasons including safety and 
efficacy, have excluded them from clinical uses (1).

Some animal studies have reported that transplantation of bone marrow stromal cells, neural progenitor cells or olfactory ensheathing cells (OECs) can promote functional improvement following SCI (27).

Olfactory ensheathing cells (OECs) are considered as a specialized population of glial cells of the primary olfactory system which are existed in both PNS and CNS $(8,9)$.

They ensheath olfactory axons in the olfactory bulb (OB), the region of termination of the olfactory sensory axons in the CNS, as well as the olfactory mucosa of the nasal cavity(9), hence the olfactory system has unique properties which consists of both PNS and CNS tissue and it is known, for its capacity, to support neurogenesis throughout life (10).

OECs have the unique property of enfolding olfactory axons along the whole axonal path from the olfactory mucosa to the outer layer of olfactory bulb and prevention of their exposures to inhibitory molecules (11). These non-myelinating glial cells play a role in guiding the axons that grow from the olfactory mucosa through the lamina propria to the OB. Therefore, they have properties of both astrocytes and Schwann cells (8, 12-14) owing to their ability to live within the $\mathrm{CNS}$ as well as axonal growth. So OECs have been suggested for cellular therapy in the nervous system, mainly for spinal cord lesions due to their properties to support axonal growth (12-14).

Functional improvement has been reported after transplantation of lamina propria into the whole thoracic spinal (15). Transplantation of olfactory mucosa in clinical trials has revealed interesting results (16). Several studies indicated that transplanted OECs could experimentally myelinate, in the right environment, the demyelinated axons $(13,17)$.

$\mathrm{Lu}$ et al and Plant et al, reported an improvement of locomotor function following delayed transplantation of OECs into the complete or incomplete thoracic spinal lesions $(15,18)$.

Preclinical studies exhibited that OEC's transplantation following spinal cord injury, such as corticospinal tract lesions, were associated with myelinating regrowing axons and functional improvements in the locomotor abilities (19).

Human OECs have been reported to remyelinate spinal cord injuries in rodents after transplantation, and accordingly in clinical trial autologous OECs were transplanted in the injured spinal cord of paraplegic humans (20).

OECs are able to stimulate tissue sparing and neuroprotection, increase outgrowth of both intact and injured axons, activate angiogenesis and remyelinte the demyelinating axons (21).

According to the previous reports, other advantages of using OECs are as follow: first, there is evidence that OEC's transplantation reduces the proteoglycans expression in the reactive astrocytes after photochemical lesions to spinal cord (22, 23). Second, OEC's reduce sprouting at the lesion area but increase the axonal growth into the distal part of transected spinal cord (24). It is believed that the role of OECs in the olfactory system is to direct the newly generated axons to their correct position in the CNS environment of the olfactory bulb (10). Third, it seems the growth factors produced by OECs play important roles in CNS regeneration. In vitro cultured rats' OECs could express NGF, GDNF, BDNF and their receptors. NGF and BDNF are important factors for survival and regrowth of damaged neurons. In addition, NGF, BDNF and PLP are the growth factors of OECs which are known to support neurite outgrowth (25).

In the other hand, OECs of the olfactory mucosa can be obtained by simple biopsy through the external nares in all individuals without affecting the smell sensation. OECs from olfactory mucosa (OM-OECs) represent a promising 
candidate for autologous transplantation to avoid graft rejection, in the nervous system specialy for spinal cord repair $(26,27)$.

In addition, mitotic activity rate and migration OECs from the lamia propria of olfactory mucosa (OM-OECs) is higher than OECs from the OB-OECs (28). Therefore, the mentioned advantages of OM-OECs prompt us to choose OM-OECs for study. So in this research OECs were separated from olfactory mucosa of 7 days old rats' pups and cultured, followed by immunolabeling for p75 and GFAP markers of OECs, and then their purity were examined by flow-cytometry after simultaneous double staining for p75 and GFAP markers.

\section{Materials and methods}

OECs culture: All cultures have been performed in the sterile conditions with autoclaved all surgery tools. Primary cultures of OM-OECs were prepared as described previously with slight modifications (29). Briefly, 7 days old wistar rats' pups were deeply anesthetized by ketamine /Xylazine $(60 / 6 \mathrm{mg} / \mathrm{Kg})$ and the nasal cavity was opened sagittally. Care was taken to avoid taking nasal septal cartilage. The $\mathrm{OM}$ of the rat is easily identified by its one-third posterior position on the nasal septum and by yellowish appearance of the epithelial surface (27). Respiratory mucosa was carefully removed from the post-anterior part of the nasal septum. After several times washing in the PBS with and then without $1 \%$ penicillin/streptomycin samples from each rat were digested enzymatically in $1 \mathrm{ml}$ of trypsin $1 \%$ for 45 min at $37^{\circ} \mathrm{C}$. Trypsinization was stopped by adding Dulbecco's Modified Eagle's/Ham's F12 medium (D.M.E.M/F12, Invitrogen), supplemented with 10\% Fetal Bovine Serum (F.B.S, Invitrogen) and $1 \%$ penicillin/streptomycin (Invitrogen) (DF-10S). Then the olfactory epithelium of $\mathrm{OM}$ was removed and underneath lamina properia was triturated and cut in some small pieces using a micropipette, until a homogenous soup (cell suspension) was obtained .The obtained soup was centrifuged at $1200 \mathrm{rpm}$ for 10 minutes, followed by resuspention in DF-10S and the centrifugation was repeated again. Then, cells were plated in $25 \mathrm{~cm}^{2}$ flasks pre-coated with poly-LLysine $(50 \mathrm{mg} / \mathrm{ml}$, Invitrogen) containing $5 \mathrm{ml}$ of DF-10S plus $2 \mu \mathrm{M}$ of forskoline mitogenic factor $(30,31)$ and $1 \%$ penicillin/streptomycin. The flasks were incubated at $37{ }^{\circ} \mathrm{C}, 5 \% \mathrm{CO} 2$.

Most of the fibroblasts and astrocytes were attached to the flask surface after $24 \mathrm{~h}$ and 48 hours respectively, while OECs remained unattached (32). Therefore, after $72 \mathrm{~h}$ OECs were collected from the supernatant and replated onto another flask and were left to grow to confluency for 14 days . The medium was changed daily.

Immunocytochemistry (ICC) of OECs: Some markers used for identification of OECs are p75, GFAP and S100 (33-35).

To determine OECs by p75 and GFAP markers in the culture, additional cell culturing was performed on cover slips pre-coated with poly-L-Lysine $(50 \mathrm{mg} / \mathrm{ml}$, Invitrogen) and was processed for immunocytochemistry. The simultaneous double immunostaining was performed for p75/GFAP, and only double positive cells in these staining were considered as OECs (33-35). The primary antibodies used were as follow: anti-p75 (Abcam,rabbit, 1:200) and anti-GFAP (Abcam ,mouse, 1:200).

The secondary antibodies used for the immunostaining were Alexa flour488 goat anti mouse and Alexa flour 568 goat anti rabbit (Abcam, 1:1000 for both).

The procedure briefly is described as follows: $5 \times 10^{2}$ cells were cultured on the cover slips pre-coated with poly-L-Lysine (50 $\mathrm{mg} / \mathrm{ml}$, Invitrogen) for $72 \mathrm{~h}$. Cell cultures were primary fixed in fresh $4 \%$ phosphate-buffered paraformaldehyde for 2 minutes. Then a secondary fixation was carried out in fresh $2 \%$ phosphate-buffered paraformaldehyde for 10 minutes at ambient temperature, followed by fixative washing and incubating with triton X-100 
for 10 minutes to cells become permeable to primary antibodies. Nonspecific binding was minimized with goat serum $10 \%$ for $1 \mathrm{~h}$ followed by incubation with primary antibodies overnight at room temperature. Secondary antibodies were incubated for $1 \mathrm{~h}$ at room temperature in dark.

Cells were counterstained for $5 \mathrm{~min}$ by DAPI $(1 \mu \mathrm{g} / \mathrm{ml})$ in dark. Then the samples were washed three times with PB (.1 M, $\mathrm{PH}=7.4)$ and one time with Diionized water and then were mounted with glycerol buffer and were observed by fluorescent microscope (Olympus AX70). It should be noted that all steps after secondary antibodies, incubation was performed in dark. Between each step, cover slips were washed tree times in PB (.1M, PH=7.4) for 10 minutes. Additionally, negative controls were stained in parallel but primary antibodies were omitted. Double positive cells for p75/GFAP were considered as OECs.

Purity evaluation of cultured OECs: For purity determining of cultured OECs Flow-cytometry was carried out. Simultaneous double staining for p75/GFAP was performed. The primary antibodies used for this procedure were anti-p75 (Abcam, rabbit, 1:200) and antiGFAP (Abcam, mouse, 1:200).

The secondary antibodies used were as follow: Alexa flour488 goat anti mouse and Alexa flour 568 goat anti rabbit (Abcam, 1:1000 for both).

The cells were washed with PB (.1 M, $\mathrm{PH}=7.4)$ and treated with trypsin/EDTA. Then, cells were washed with $\mathrm{PB}$ and double immunolabeled, during $1 \mathrm{~h}$ at ambient temperature, with the antip75/anti-GFAP. At the end of incubation, $2 \mathrm{ml}$ of $\mathrm{PB} .1 \mathrm{M}(\mathrm{PH}=7.4)$ was added and the cells were centrifuged (1200rpm for 10 min). Cells were incubated with the secondary antibodies $(1: 1000)$ for $1 \mathrm{~h}$ in the dark. At the end of the incubation, $2 \mathrm{ml}$ of PB .1 M (PH=7.4) was added and the cells were centrifuged (1200rpm for $10 \mathrm{~min}$ ). Then, cells were resuspended in $1 \mathrm{ml}$ of PB (.1 M, PH=7.4). The total number of 10,000 events was analyzed on a flowcytometer (FACS, USA) directly after the staining procedure. The experiments were triply repeated and the results were expressed as mean \pm standard deviation (SD).

\section{Statistical analysis}

Statistical analysis was performed using MiniTab software (release 11.12, Minitab Inc., State College, PA, USA). The experiments were all repeated three times and the results reported as mean \pm standard deviation (SD) at a significance level of $\mathrm{p} \leq 0.05$. Differences were considered statistically significant when the $\mathrm{p}$ value was equal or less than 0.05 .

\section{Results}

As Figure 1 indicates the cultured OECs demonstrated two different phenotypes after attaching to flask surface, including a spindle shape Schwann-like and an astrocyte-like OECs with flat sheet-like morphology In addition, simultaneous immunolabeling for p75 and GFAP markers of OECs demonstrated OECs positivity for both markers at the same time (Figure 2).

Furthermore, the flow-cytometry results displayed $87.9 \pm 2.4 \%$ of cells were p75/ GFAP double positive cells, $1.05 \pm .4$ only p75 positive and $5.8 \pm 1.5 \%$ were single positive for GFAP ( Figure 3). The experiments for flow-cytometry were repeated three times and the results were demonstrated as mean \pm standard deviation (SD). 


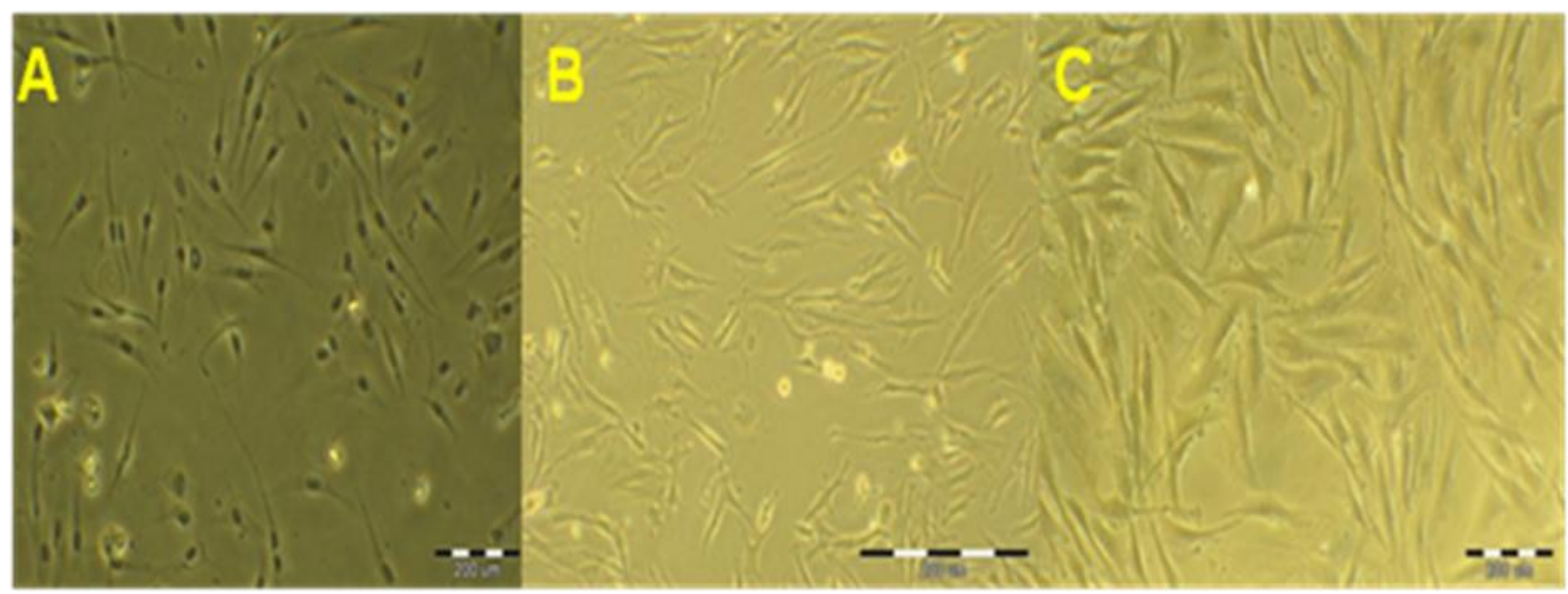

Figure 1. Phase-contrast images of primarily cultured OECs for 10 days. Both morphology of cultured OECs defined as Schwann-like and Astrocyte-like (fibroblast like): A) Schwann-like with a long fusiform bipolar morphology, B and C) both types of OECs observed with different magnifications. Astrocyte-like with a flat sheet-like morphology and short randomly oriented processes.

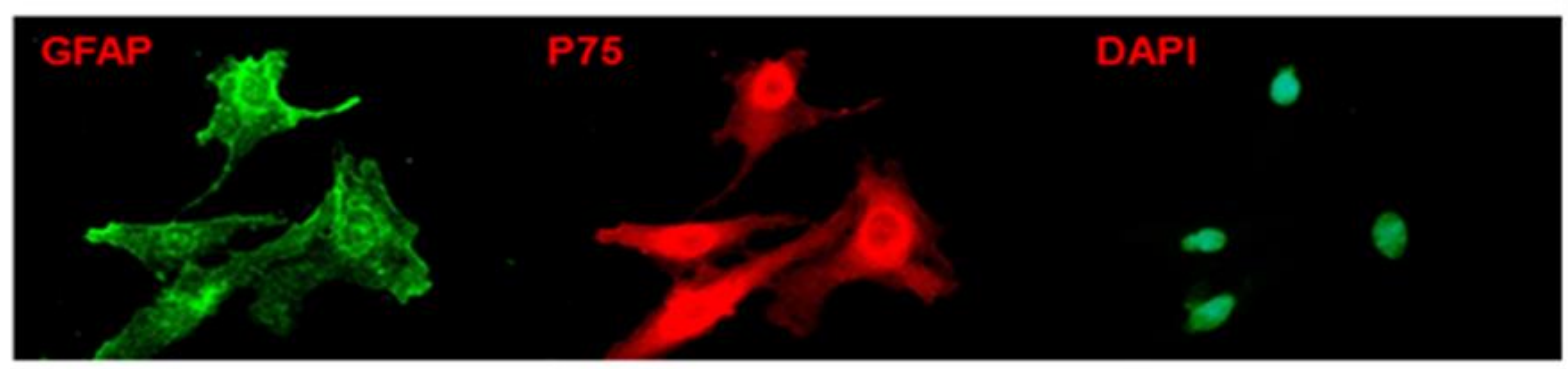

Figure 2. Simultaneous double staining (Immunocytochemistry (ICC)) for p75 and GFAP markers of cultured OECs , p $75^{+} / \mathrm{GFAP}^{+}$are double positive cells for p75 and GFAP markers at the same time. Counterstaining (nuclear staining) was performed by DAPI in all samples.

\section{Discussion}

Cell transplantation is one of the most popular strategies for SCI repair. So, the main objective of the current research was OECs purifying in the primary cultures by simple method and determining their purity. Hence, OECs were isolated from olfactory mucosa (OM) of 7 days old wistar rats' pups and were cultured. For determining of OECs and their purity Immunocytochemistry and Flowcytometry were performed respectively. Cultured OEC's by our team demonstrated two different phenotypes after attaching to flask surface which was in accordance with other researchers who also reported two subpopulations of OEC's $(31,36)$.

Barnet and Riddle exhibited two subtypes of OEC's that were purified from the olfactory bulb of 7-day old SpragueDawly rats' pups after 12 days that was in agreement with our findings (37).

Ensheathing cell morphology is categorized as flat or process-bearing. Flat OEC's (schwann-like) characterized by their long fusiform bipolar morphology (35). Process-bearing (Astrocyte/or fibroblast-like) OEC's are identified with very little cytoplasm and some fine randomly oriented processes longer than the width of the cell body (35) .Vincent and coworkers proposed that the morphological plasticity of OEC's is related to the culture conditions and is rapid and reversible (35). 

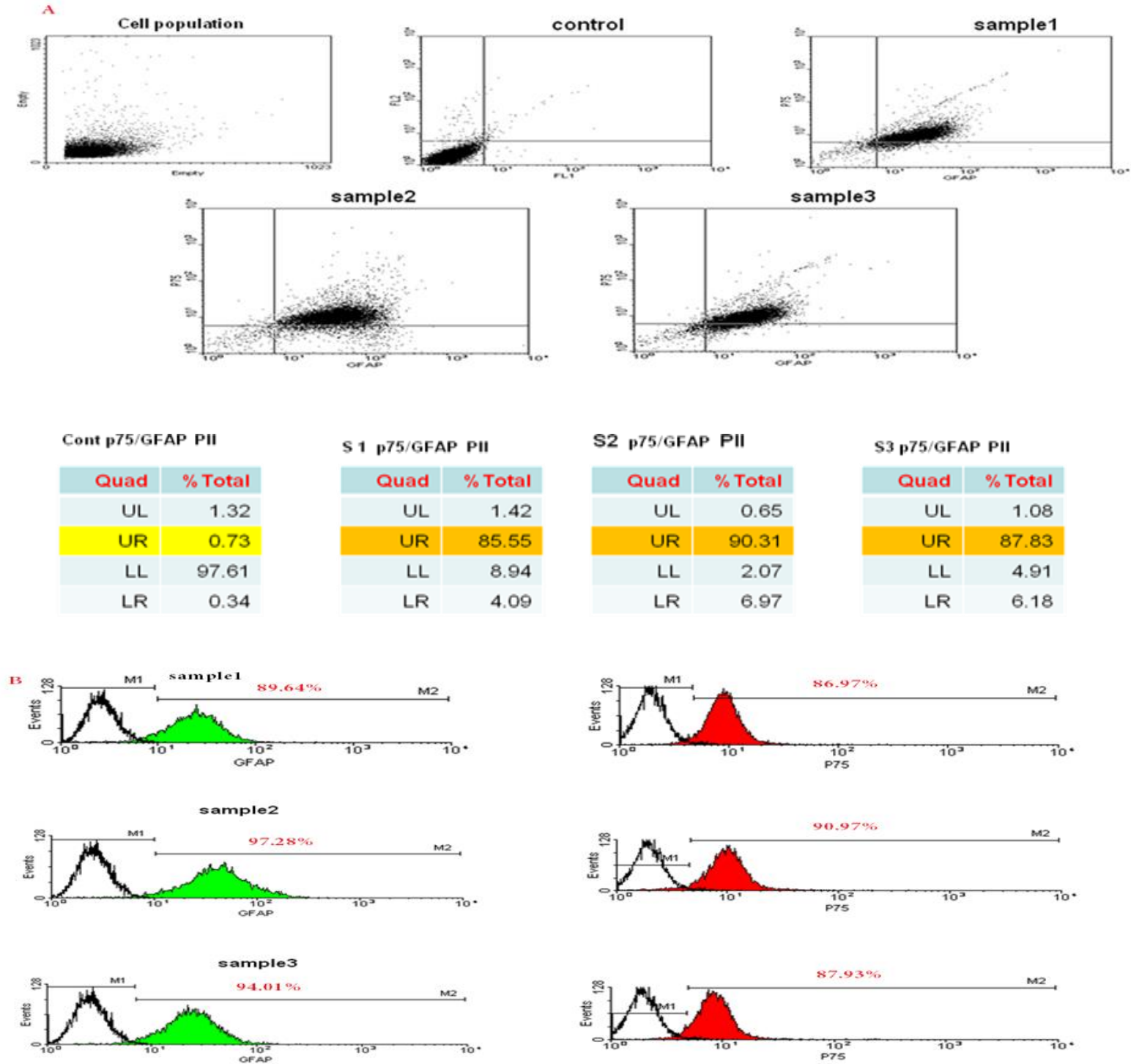

Figure 3. Cell population graph, A) Dot Plot, B) Histogram from flow-cytometry of OECs for p75/GFAP markers after second passage of cultured cells. In the Dot plot UR demonstrates cells that were double positive for two markers simultaneously, but UL and LR demonstrate cells that were single positive for one marker only. Experiment was performed three times (sample1,2,3) to confirm it's reproducibility and results were expressed as mean $\pm \mathrm{SD}$. Control group (Negative control) was the group in which primary antibodies were omitted and were stained in parallel.

Vincent and his research group showed that cultured OEC's undergo rapid and reversible changes between flat morphology in the presence of serum and process-bearing morphology in the absence of serum (35).

Purity of OEC's was examined after simultaneous double staining for $\mathrm{p} 75 /$ GFAP, by flow-cytometry. The cell markers p75 and GFAP were chosen to identify OEC's owing to no coexpression of these proteins by other cell types from the olfactory mucosa. Only cells, immunopositive for both markers were defined as OEC's (35). Our flowcytometry results showed that $87.9 \pm 2.4 \%$ of cells were p75/ GFAP double positive cells, $1.05 \pm 0.4$ only p75 positive and $5.8 \pm 1.5 \%$ were single positive for GFAP only. But p75+/GFAP+ and p75+/S100+ or GFAP $+/ \mathrm{S} 100+$ were olfactory ensheathing cells. In the culture procedure used in this study, $87.9 \%$ of cells were the population of p75+/GFAP+ OEC's alone. Thus purity of our culture was probably more than $87.9 \%$, because some of single 
p75 and GFAP positive cells (p75+ and GFAP+) in this immunostaining could be as p75+/S100+ and GFAP+/S100+ OEC's. Hence triple immunolabeling for these three markers of $\mathrm{OEC}^{\prime}$ was more efficient to complete determining of OEC's purity, amounts of astrocytes and other cells which could contaminate our culture. Schwann cells from the trigeminal nerve are considered as bipolar p75-positive and GFAP-negative and cells that labeled strongly with GFAP but not p75-positive (p75-/ GFAP+) are considered as astrocyte. Astrocytes are abundant in the olfactory nerve layer (ONL) culture whereas they are absent in the OM culture (35).

In agreement with our results, there are findings of investigators that reported the percentage of OM-OEC's after single labeling for p75 marker by flow-cytometry for $85 \%$ (38).

But these investigators, in another report, reported that approximately $10 \%$ of OMOEC's were p75-positiveby immunocytochemistry and flow-cytometry (39).

Another research by Vincent and his coworkers cultured OEC's from both olfactory mucosa (OM) and olfactory nerve layer (ONL), in unpurified separated cultures for 2 days in serum-containing Dval-10dS medium and reported that in both cultures, up to $70 \%$ of OEC's were process-bearing (astrocyte-like) and 30\% were flat(Schwann-like), and both morphologies displayed GFAP staining. In this study p75/GFAP -positive cells were approximately $14 \%$ of total population in

\section{References}

1. Li J, Lepski G. Cell transplantation for spinal cord injury: A systematic review. Biomed Res Int. 2013; 2013:786475.

2. Hernandez J, Torres-Espin A, Navarro X. Adult stem cell transplants for spinal cord injury repair: current state in preclinical research. Curr Stem Cell Res Ther. 2011; 6(3):273-87. the $\mathrm{OM}$ culture and approximately $6 \%$ in the olfactory nerve layer (35). Some possible explanations that account for this discrepancy probably are: the time difference (2 days in Vincent study and 14 days in our study) and method and supplement used for OEC's culture as well as kind of the technique used for purity assessment (imunolabeling in Vincent study but flow-cytometry in our study).

The different experimental procedures used by investigators to purify and culture OEC's seem to partially contribute the varied descriptions of this cell type (35). Additionally, in the culture, the proportion of each subtype changes with the length of culture time (31).

\section{Conclusion}

Purity of cultured OECs for p75+/GFAP+ cells of the culture was $87.9 \pm 2.4 \%$ by flow-cytometry and it's percentage may be more than this if the p75+/S100+ and GFAP+/S100+ cells were immunostained and counted. Thus the culture procedure of this study seems to be a good protocol for OEC's purifying and cell therapy.

\section{Acknowledgments}

This research was supported by a grant from the Iran National Science Foundation (INSF) (no. 89003650). The authors would like to thank Anatomy Department and Cellular - Molecular research center of Iran University of Medical Sciences for their technical support. We also thank Atousa Hayat and Maryam Soleimani for their helpful assistance in flow-cytometry.

3. Das AK, Gopurappilly R, Parhar I. Current status and prospective application of stem cell-based therapies for spinal cord injury. Curr Stem Cell Res Ther. 2011; 6(2): 93-104.

4. Kan EM, Ling EA, Lu J. Stem cell therapy for spinal cord injury. Curr Med Chem. 2010; 17(36): 4492-10. 
5. Centenaro LA, Jaeger C, Ilha J, de Souza MA, Kalil-Gaspar PI, Cunha NB, et al. Olfactory and respiratory lamina propria transplantation after spinal cord transection in rats: effects on functional recovery and axonal Regeneration. Brain Res. 2011; 1426: 54-72.

6. Zhang SX, Huang F, Gates M, White J, Holmberg EG. Histological repair of damaged spinal cord tissue from chronic contusion injury of rat: a LM observation. Histol Histopath.2011; 26(1): 45-58.

7. Zhang SX, Huang $F$, Gates $M$, Holmberg EG. Scar ablation combined with LP/OEC transplantation promotes anatomical recovery and $\mathrm{P} 0$-positive myelination in chronically contused spinal cord of rats. Brain Res. 2011; 1399: 1-14.

8. Ramón-Cueto A, Avila J. Olfactory ensheathing glia: properties and function. Brain Res Bull.1998; 46(3): 175-87.

9. Vincent AJ, West AK, Chuah MI. Morphological and functional plasticity of olfactory ensheathing cells. J Neurocytol. 2005; 34(1-2): 6580.

10. Doucette R. Glial influences on axonal growth in the primary olfactory system. Glia. 1990; 3(6):433-49.

11. Pasterkamp RJ, De Winter F, Holtmaat AJ, Verhaagen J. Evidence for a role of the chemo repellent Semaphorin III and its receptor Neuropilin-1 in the regeneration of primary olfactory axons. J Neurosci. 1998; 18(23): 996276.

12. Li Y, Field PM, Raisman G. Regeneration of adult rat corticospinal axons induced by transplanted olfactory ensheathing cells. J Neurosci. 1998; 18(24): 10514-24.

13. Imaizumi $\mathrm{T}$, Lankford KL, Waxman SG, Greer CA, Kocsis JD. Transplanted olfactory ensheathing cells remyelinate and enhance axonal conduction in the demyelinated dorsal columns of the rat spinal cord. J Neurosci. 1998; 18: 6176-85.

14. Ruitenberg MJ, Vukovic J, Sarich J, Busfield SJ, Plant GW. Olfactory ensheathing cells: Characteristics, genetic engineering, and therapeutic potential. J Neurotrauma. 2006; 23(34): 468-78.

15. Lu J, Feron F, Mackay-Sim A, Waite PM. Olfactory ensheathing cells promote locomotor recovery after delayed transplantation into transected spinal cord. Brain. 2002; 125(1): 1421.

16. Lima C, Pratas-Vital J, Escada P, Hasse-ferreira A, capucho C, Peduzzi J. Olfactory mucosa autograft in human spinal cord injury: A pilot clinical study. J Spinal Cord Med. 2006; 29(3): 191-203.

17. Sasaki M, Lankford KL, Zemedkun M, Kocsis JD. Identified olfactory ensheathing cells transplanted into the transected dorsal funiculus bridge the lesion and form myelin. $\mathrm{J}$ Neurosci. 2004; 24(39): 8485-93.

18. Plant GW, Christensen CL, Oudega $\mathrm{M}$, Bunge $\mathrm{M}$ B. Delayed transplantation of olfactory ensheathing glia promotes sparing/regeneration of supra spinal axons in the contused adult rat spinal cord. J Neurotrauma. 2003; 20(1): 116.

19. Mackay-Sim A. Olfactory ensheathing cells and spinal cord repair. Keio $\mathbf{J}$ Med. 2005; 54(1): 8-14.

20. Feron F, Perry C, Cochrane J, Licina P, Nowitzke A, Urquhart $\mathrm{S}$, et al. Autologous olfactory ensheathing cell transplantation in human spinal cord injury. Brain. 2005; 128(12): 2951-60.

21. Kocsis JD, Lankford KL, Sasaki M, Radteke C. Unique in vivo properties of olfactory ensheathing cells that may contribute to neural repair and protection following spinal cord injury. neurosci let. 2009; 456(3):137-42.

22. Verdu E, Garcia-Alias G, Fores J, Gudino-Cabrera G, Muneton VC, 
Nieto- Sampedro M, et al . Effects of ensheathing cells transplanted into photo chemically damaged spinal cord. Neuroreport. 2001; 12(11):2303-9.

23. Verdu E, Garcia-Alias G, Joaquimfore S, Lopez-Vales R, Navarro $\mathrm{X}$. Olfactory ensheathing cells transplanted in Lesion spinal cord prevent loss of spinal cord parenchyma and promote functional recovery. Glia. 2003; 42(3):275-86.

24. Li Y, Decherchi P, Raisman G. Transplantation of olfactory ensheathing cells into spinal cord lesions restores breathing and climbing. Neuroscience. 2003; 23(3):727-31.

25. Emma W, Adrian KW, Meng IC. Cultured olfactory ensheathing cells express growth factor, brain-derived neurotrophic factor, glia cell linederived neurotrophic factor and their receptors. Mol Brain Res. 2001; 88(12):203-13.

26. Feron F, Perry C, McGrath JJ, Mackay-Sim A. New techniques for biopsy and culture of human olfactory epithelial neurons. Arch Otolaryngol Head Neck Surg. 1998; 124(8): 861-6.

27. Lu J, Feron F, Ho SM, Mackay-Sim A, Waite PM. Transplantation of nasal olfactory tissue promotes partial recovery in paraplegic adult rats. Brain Res. 2001; 889(1-2): 344-57.

28. Richter MW, Fletcher PA, Liu J, Tetzlaff W, Roskams AJ. Lamina propria and olfactory bulb ensheathing cells exhibit differential integration and migration and promote differential axon sprouting in the lesion spinal cord. J Neurosci.2005; 25(46):10700 11.

29. Jani HR, Raisman G. Ensheathing cell cultures from the olfactory bulb and mucosa. Glia. 2004; 47(2): 130-7.

30. Takami T, Oudega M, Bates ML, Wood PM, Kleitman N, Bartlett MB. Schwann cell but not olfactory ensheathing glia transplants improve hindlimb locomotor performance in the moderately contused adult rat thoracic spinal cord. J Neurosci. 2002; 22(15): 6670-81.

31. Huang Z, Wang Y, Cao L, Su Z, Zhu Y, Chen Y, et al. Migratory properties of cultured olfactory ensheathing cells by single-cell migration assay. Cell Res. 2008; 18(4):479-90.

32. Wang B, Zhao $\mathrm{Y}$, Lin $\mathrm{H}$, Chen B, Zhang J, Wang $X$, et al. Phenotypical analysis of adult rat olfactory ensheathing cells on 3-D collagen scaffolds. Neurosci Let. 2006; 401(12): 65-70.

33. Cao L, Liu L, Chen ZY, Wang LM, Ye JL, Qiu HY, et al. Olfactory ensheathing cells genetically modified to secrete GDNF to promote spinal cord repair. Brain. 2004; 127(3): 53549.

34. $\mathrm{Au}$ E, Roskams AJ. Olfactory ensheathing cells of the lamina propria in vivo and in vitro. Glia. 2003; 41(3):224-36 .

35. Vincent AJ, West AK, Chuah MI. Morphological plasticity of olfactory ensheathing cells is regulated by cAMP and endothelin-1.Glia. 2003; 41(4):393-403.

36. Alexander CL, Fitzgerald UF, Barnett SC. Identification of growth factors that promote long-term proliferation of olfactory ensheathing cells and modulate their antigenic phenotype. Glia. 2002; 37(4):349-64.

37. Barnet SC, Riddle JS. Olfactory ensheathing cells (OECs) and the treatment of CNS injury: advantages and possible caveats. J Anat. 2004; 204:57-67 .

38. Guerout N, Drambure C, Drouot L, Bon-mardion N, Duclos C, Boyer O, et al. Comparative gene expression profiling of olfactory ensheathing cells from olfactory bulb and olfactory mucosa. Glia. 2010; 58(13):1570-80.

39. Guerout N, Paviot A, Bon-Mardion N, Duclos C, Genty D, Jean L, et al. Cotransplantation of olfactory ensheathing cells from mucosa and 
bulb origin enhances functional PLoS One. 2011;6(8):e22816. recovery after peripheral nerve lesion. 